\title{
In Situ Melanoma in Collision With a Basal Cell Carcinoma in a Patient With Basal Cell Nevus Syndrome: Clinical and Dermoscopic Features
}

\author{
Giulia Briatico ${ }^{1}$, Elvira Moscarella ${ }^{1}$, Andrea Ronchi ${ }^{2}$, \\ Enrico Maria Procaccini ${ }^{1}$, Giuseppe Argenziano ${ }^{1}$
}

\begin{abstract}
1 Dermatology Unit, University of Campania, Luigi Vanvitelli, Naples, Italy
\end{abstract}
2 Pathology Unit, University of Campania, Luigi Vanvitelli, Naples, Italy

Key words: basal cell carcinoma, melanoma in situ, basal cell nevus syndrome, collision tumors

Citation: Briatico G, Moscarella E, Ronchi A, Procaccini EM, Argenziano G. In situ melanoma in collision with a basal cell carcinoma in a patient with basal cell nevus syndrome: clinical and dermoscopic features. Dermatol Pract Concept. 2019;9(4):310-312. DOI: https://doi. org/10.5826/dpc.0904a16

Accepted: April 25, 2019; Published: October 31, 2019

Copyright: $\odot 2019$ Briatico et al. This is an open-access article distributed under the terms of the Creative Commons Attribution License, which permits unrestricted use, distribution, and reproduction in any medium, provided the original author and source are credited.

Funding: None.

Competing interests: The authors have no conflicts of interest to disclose.

Authorship: All authors have contributed significantly to this publication.

Corresponding author: Elvira Moscarella, MD, University of Campania, Luigi Vanvitelli, Dermatology Unit, Via Sergio Pansini, 5, Naples, 80121, Italy. Email: Elvira.moscarella@gmail.com

\section{Introduction}

Basal cell nevus syndrome (BCNS), also known as Gorlin-Goltz syndrome, is a rare autosomal dominantly inherited disorder characterized by the development of basal cell carcinomas (BCCs) from a young age, multiple keratocysts, palmar and/or plantar pits, calcification of falx cerebri, and family aggregations. Other criteria are skeletal anomalies, frontal bossing, cardiac and ovarian fibromas, medulloblastoma, glaucoma, and cleft lip/palate.

The disorder is caused by an alteration of the sonic hedgehog signaling pathway, which results in constitutive activity and tumor cell proliferation. Some germline pathogenic variants of these genes, including PTCH1 and SUFU, have been found and are responsible for clinical heterogeneity.

Only 2 case reports have described the occurrence of melanoma in patients with BCNS, but neither reported the occurrence of collision lesions [1,2]. Here we present clinical, dermoscopic, and histological features of a collision tumor of BCC and in situ melanoma in a patient with multiple BCCs and BCNS.

\section{Case Presentation}

A 48-year-old man came to our attention for multiple BCCs of the face, arms, and trunk. Family history revealed a sister with the genetic diagnosis of BCNS and a history of ovarian fibromas and keratocysts. One brother with a cleft palate died at birth.

The patient suffered from multiple kidney cysts, glaucoma, and scoliosis. At birth, he received the diagnosis of macrocephaly. Palmar or plantar pitting was not evident.

Clinical examination revealed multiple lesions suggestive of BCCs on the trunk. The patient also had multiple nevi. We observed an asymmetric partially pigmented lesion on 
the lower back, approximately $1 \mathrm{~cm}$ in diameter, with a central amelanotic nodular component.

Upon dermoscopy, the lesion was asymmetrical, displaying a pink structureless area in the center with short, fine telangiectasias and an atypical network at the periphery (Figure 1).

Histological examination showed a combined neoplasm. On one side, a proliferation of atypical melanocytes organized in a lentiginous pattern consistent with in situ melanoma was evident. On the other side, we observed a nodular BCC (Figure 2).

\section{Conclusions}

Collision tumor of BCC and melanoma is an uncommon but well-described event. Several cases of clinical and dermoscopic features of melanomas colliding with BCCs have been reported in the literature. In fact, BCC is the more represented malignant tumor among collision lesions, simply because of its

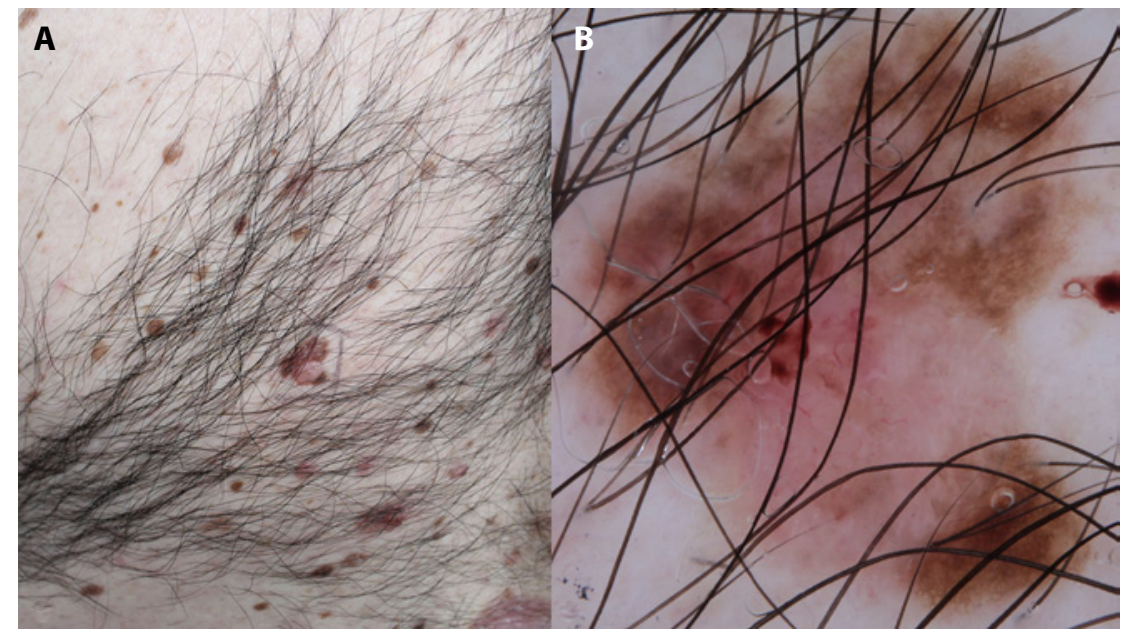

Figure 1. (A) Clinical appearance of the back of the patient, a 48 -year-old man, showing multiple nevi and 1 asymmetrically pigmented lesion with a central amelanotic nodular component and brown pigmentation in the periphery. (B) Dermoscopy, showing a pink structureless area in the center with short, fine telangiectasias and an atypical network at the periphery. [Photograph by Dermaview Tre T Medical. Copyright: @2019 Briatico et al.]

high prevalence in the Caucasian population. When colliding with melanoma, the nodular component of a BCC could be misdiagnosed as a nodular component of the melanoma.

In our case, the patient's history of multiple BCCs in the context of
BCNS prompted us to look dermoscopically at all lesions of the patient. We considered the development of melanoma in our patient as circumstantial, because melanoma and Gorlin-Goltz syndrome have different molecular pathogeneses.

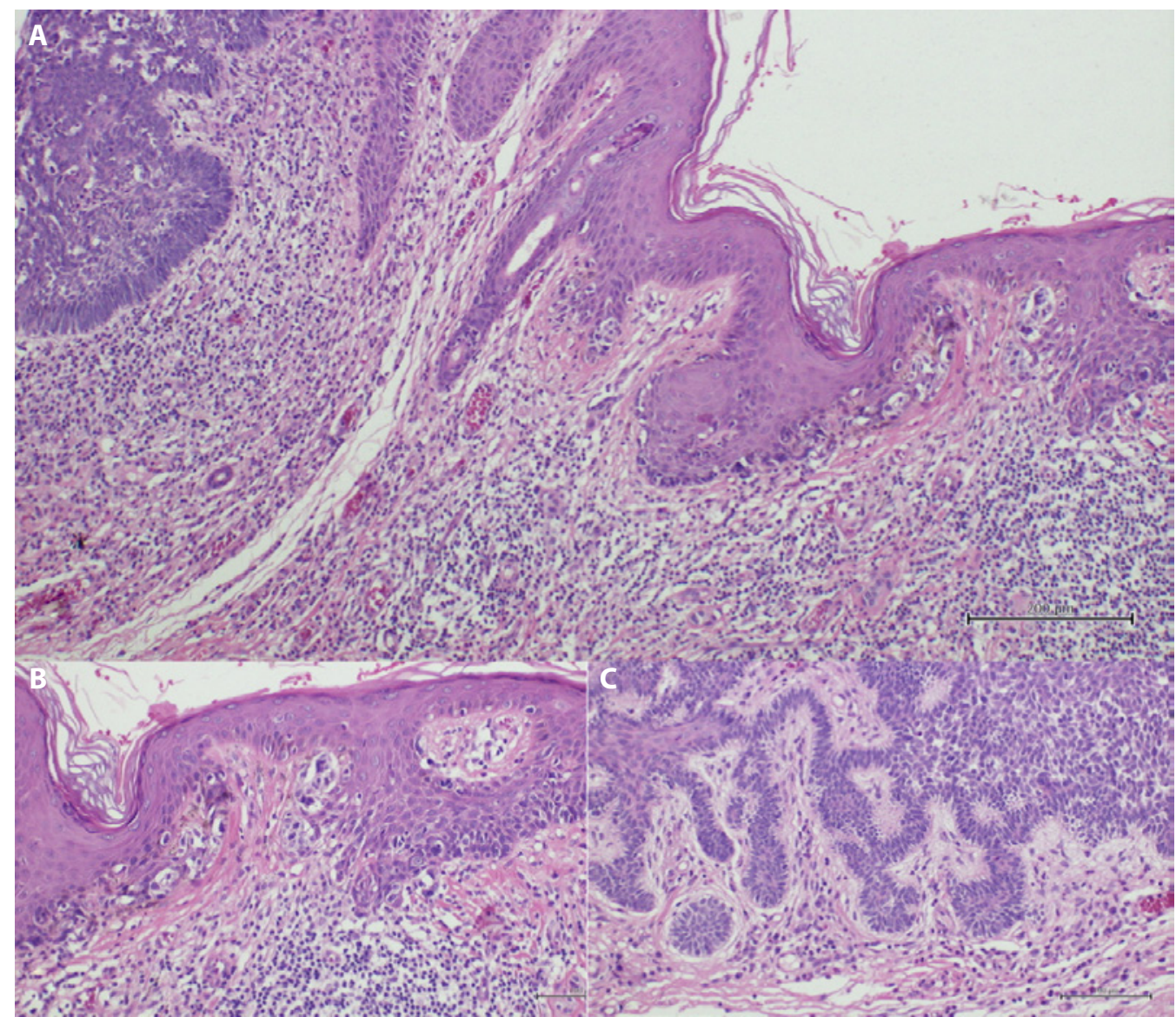

Figure 2. (A) Histological examination shows a combined neoplasm. (B) In detail, on one side, a proliferation of atypical melanocytes organized in a lentiginous pattern consistent with in situ malignant melanoma is evident. (C) On the other side, we see a nodular basal cell carcinoma (H\&E, magnification $\times 10)$. [Copyright: (C2019 Briatico et al.] 
This case underlines once more the importance of total body clinical and dermoscopic examination. In situ melanoma in collision with BCC in BCNS is an exceptional event; however, an accurate dermoscopic examination may serve to exclude concomitant malignancies, especially in view of systemic treatment for BCCs.

\section{References}

1. Gregoriou S, Kazakos C, Belyaeva H, et al. Hypomelanotic nail melanoma in a patient with Gorlin syndrome. J Cutan Med Surg. 2012;16(2):143-144.

2. Kedem A, Even-Paz Z, Freund M. Basal cell nevus syndrome associated with malignant melanoma of the iris. Dermatologica. 1970;140(2):99-106. 